\title{
Steroid as an Intracanal Medicament: An Advanced Review
}

\author{
Priyankita Kar ${ }^{D}$ *, Rana K Varghese ${ }^{2}$, Naina Agrawal' ${ }^{2}$, Himanshi \\ Solanki Jhaveri ${ }^{2}$ \\ ${ }^{1}$ New Horizon Dental College and Research Institute, Sakri, Bilaspur, Chhattisgarh, India. \\ ${ }^{2}$ New Horizon Dental College and Research Institute, Sakri, Bilaspur, Chhattisgarh, India.
}

\section{ARTICLE INFO}

Article History

Received:May 2021

Accepted: Apr 2021

ePublished: June 2021

Corresponding author:

Priyankita Kar,New Horizon Dental College and

Research Institute, Sakri,

Bilaspur, Chhattisgarh,

India. Email: dr.priyankita.

kar@gmail.com

\begin{abstract}
Post-endodontic treatment pain is a major problem in dentistry. Around $80 \%$ of patients who present with preoperative pain due to endodontic treatment continue to experience the pain of some level following endodontic therapy. This pain, mostly linked to chronic inflammation, is due to bacterial toxins that signal the ingress of immune cells followed by the activation of various cytokines and inflammatory mediators. Countless classes of drugs have been studied to resolve this challenge. Glucocorticoids have been in use for more than fifty years owing to their potent antiinflammatory effects. This paper aims to review the pharmacology and mechanism of actions of steroids as an intracanal medicament considering the indications, contraindications, and side effects.
\end{abstract}

J Res Dent Maxillofac Sci 2021;6(3):47-51.

Keywords: Ledermix, Triamcinolone, Postoperative Pain, Hydrocortisone

\section{Introduction}

Chemomechanical preparation and complete disinfection of the root canal system are crucial for successful endodontic therapy. However, intracanal medicaments are indispensable means to kill the bacteria, cleaning and shaping protocols have assumed a greater reputation than the former as a mode of root canal disinfection..$^{(1)}$ In situations where the treatment cannot be rendered in a single appointment, there are probabilities that the bacteria, which have survived inside the dentinal tubules, continue to proliferate between the scheduled appointments. ${ }^{(2)}$ Walton has stated that "intracanal medicaments have traditionally gone hand-in-glove with endodontics. They are generally considered to be an integral part of treatment and important to the success of root canal therapy." To curb bacterial regrowth and develop bacterial suppression, an intracanal medication can be beneficial. The purpose of the inter-appointment antimicrobial intracanal medication is to limit the proliferation or eliminate the bacteria along with curtailing the ingress of pathogens through any leaking restorations. ${ }^{(3)}$

Chemical mediators, like bradykinin and prostaglandin, which are released from injured/ inflamed tissues, can induce pain. Corticosteroids have the biological ability to inhibit the inflammatory response, ${ }^{(2,3)}$ and therefore, can be expected to reduce or eliminate pain associated with inflamed tissues. Steroids are used efficiently to relieve pulpal and periradicular pain during and after the procedures. They are an excellent adjunct to flare-up treatments as well.

Brief physiology of steroids

Steroids, the complex lipophilic molecules, have numerous actions in the body, which include the regulation of cellular, tissue, and organ functions. All steroids in the body are derived from cholesterol via tightly regulated biosynthetic enzymatic pathways that operate predominantly in specific endocrine organs, including the adrenal glands, ovaries, and testis. ${ }^{(4)}$ The adrenal gland produces three groups of steroid hormones in the human body including:

- Androgens

- Mineralocorticoids

- Glucocorticoids

The adrenal cortex secretes glucocorticoid and other steroids, which have extensive effects on the metabolism of carbohydrates and proteins. ${ }^{(5)}$ 
Usages of steroids in dentistry

Steroids are commonly used to limit postoperative inflammation. In major surgical procedures, the main function of steroids is to decrease edema, trismus, pain, and hospitalization time. ${ }^{(6)}$ They are used in conditions like recurrent aphthous ulceration, desquamative gingivitis, lichen planus, bullous pemphigoid, mucous membrane pemphigoid, pemphigus vulgaris, and erythema multiforme. Intralesional injections are administered for the management of mucocele, oral submucous fibrosis, and central giant cell granuloma. They are also used for the management of Bell's palsy, Melkersson-Rosenthal syndrome, and temporomandibular disorders (TMDs).

To manage acute allergic reactions, corticosteroids are useful after the use of epinephrine and histamine blockers to prevent recurrent anaphylactic shock. They are used as the second line of drugs because of their slow onset of action. Because dexamethasone and methylprednisolone are contraindicated in acute adrenal insufficiency, hydrocortisone sodium succinate is the drug of choice. $^{(7)}$

Corticosteroids are used as an endodontic anodyne. ${ }^{(8)}$ Along with the broad-spectrum antibiotics, steroids are used as pulp capping agents because of their anti-inflammatory properties as follows: ${ }^{(9)}$

- Pulpovital $=$ Prednisolone + Chloramphenicol

+ Neomycin

- Dontisolon $=$ Prednisolone + Neomycin

- Septomixine $=$ Dexamethasone + Polymycin Sulfate + Neomycin

- Cavity liners = 1\% Prednisolone $+25 \%$ Chloramphenicol $+50 \%$ Gum Camphor to reduce postoperative thermal sensitivity.

Triamcinolone acetonide is a potent corticosteroid that could be used effectively to eliminate or at least reduce the severe inflammation that might occur secondary to endodontic treatment. ${ }^{(10)}$ The application of corticosteroids to exposed dentine following cavity preparation, in indirect pulp capping, to the exposed pulp or the pulp remnants and periapical tissue during root canal therapy has been claimed to eliminate postoperative pain and inflammation. ${ }^{(11)}$ A study of molecular biology concluded that dexamethasone promotes osteogenic differentiation in mesenchymal stem cells, depending on the concentration. ${ }^{(12)}$
A cohort study on postoperative pain after root canal therapy concluded that the mean incidence of post-endodontic pain was $54.7 \%$ after 6 hours and $46.4 \%$ after 18 hours. ${ }^{(13)}$ It was also evident from earlier studies that analgesic interventions are essential in some endodontic cases after treatment. Many classes of drugs have been considered and researched for the management of post-treatment endodontic pain, including opioids, non-narcotic analgesics like non-steroidal anti-inflammatory drugs (NSAIDs) and acetaminophen, and steroids. ${ }^{(14)}$

\section{Indications and contraindications}

Kawashima et al have defined intracanal medicament as "temporary placement of medicaments with good biocompatibility into root canals to inhibit coronal invasion of bacteria". Though there have been claims, no medicament is deemed to be ideal, and their use in dental practice is significantly unpredictable. ${ }^{(1)}$ A tooth with a vital pulp does not generally require an intracanal medicament. Their role is more relevant, though complex, in cases of pulpal necrosis and apical periodontitis. ${ }^{(1)}$

Properties of an ideal intracanal medicament

An ideal intracanal medicament should be an effective antimicrobial agent. It must be stable, potent in the presence of serum, blood, and protein derivatives of tissues with prolonged antimicrobial effects. It is advantageous if it has low surface tension. It must not discolor the tooth. Most importantly, no cell-mediated immune response should be induced, which could interfere with the repair of periradicular tissues.

Indications of intracanal medicaments

-In cases of weeping canals, which are persistently wet, intracanal medicament could be used to dry and heal.

-Eliminating occult pathogens inside dentinal tubules or in pulp space owing to its high alkalinity. -Helpful in rendering the root canal contents inert.

-Neutralizing the acidic $\mathrm{pH}$ due to inflammation. -In cases of symptomatic patients, it can act as a barrier against leakage during inter-appointments. ${ }^{(15)}$

Steroid as an intracanal medicament

In 1954, Wolfsohn first reported the usage of steroids as an intracanal medicament and stated that "the use of hydrocortisone helped in the re- 
duction as well as the elimination of severe secondary inflammatory reactions in the periodontal membrane following treatment". (16) Blitzer suggested that in cases where polyantibiotic therapy failed, the use of TACT [Terramycin, Antihistamine, Cortril (25mg/cc Hydrocortisone) and Tetracyn] could be beneficial. ${ }^{(17)}$ Ledermix, a popular steroid intracanal medicament, is a glucocorticosteroid-antibiotic compound. The credit of the development of Ledermix goes to Schroeder and Triadan in 1960, being released for sale in Europe by Lederle Pharmaceuticals in 1962. The sole reason for adding the antibiotic component to Ledermix was to compensate for what was perceived to be a possible corticoid-induced reduction in the host immune response (18) $^{(18)}$

Ledermix, which has two therapeutic components (triamcinolone and demeclocycline), can diffuse through dentinal tubules and cementum to reach the periodontal and periapical tissues ${ }^{(19)}$ It has been used for pulp capping, pericementitis, and patients who experienced constant postoperative pain following pulp extirpation or root canal instrumentation. $^{(20)}$

According to a systematic review and metaanalysis by Shamszadeh et al, ${ }^{(21)}$ dexamethasone and betamethasone are long-acting steroids with a biological half-life between 36 and 72 hours, whereas prednisolone is an intermediate-acting steroid with a half-life of 200 minutes. ${ }^{(22)}$ The shorter half-life of prednisolone, as compared to other drugs, could be a reason for its higher efficacy. This could be explained by the fact that the intensity of postoperative endodontic pain decreased over time. ${ }^{(19)}$ The results showed that the dose of the drug might have influenced the rate of pain reduction provided by corticosteroids. This could be because greater pain reduction was seen in trials investigating corticosteroids with a higher dose at 6 and 12 hours postoperatively. As endodontic pain reduced over time, the rationale that the higher dosages of corticosteroids did not influence the treatment effects at 24 hours stands effective.

Triamcinolone is gradually released by diffusion through the dentinal tubules and cementum, finally reaching the periapical and periodontal tissues. ${ }^{(23)}$ Thirty percent of it is released after the first 24 hours, and the remaining 70\% is released by the end of 14 weeks. Zanjir et al (2020), in their systematic review and network meta-analysis, stated that corticosteroids were ineffective in cases of necrotic pulp and did not reduce pain, which contradicted some earlier studies. They also concluded that steroids could induce immunosuppression at high doses and longer durations. Therefore, they should be considered only when other anti-inflammatory drugs have been proven useless. ${ }^{(24)}$

Combination of Ledermix and calcium hydroxide

Schroeder advocated the use of Ledermix in conjunction with calcium hydroxide for the management of necrotic teeth with an open apex. Ledermix paste and calcium hydroxide (1:1) have also been used to disinfect root canals and to treat pulp necrosis, as well as for apexification of open apices and treatment of tooth perforations, large periapical lesions, inflammatory root resorption, and inflammatory periapical bone resorption. ${ }^{(18)}$ A mixture at this ratio of active components allows slow release and diffusion, making the medicament last longer in the canals. ${ }^{(18)}$ This, in turn, aids in maintaining the sterility of the canal for a long period along with maintaining higher concentrations of all components within the canal. It acts similar to when calcium hydroxide is used alone as it does not alter the $\mathrm{pH}$ significantly. Taylor et al demonstrated that for two indicator cariogenic microorganisms, Lactobacillus casei and Streptococcus mutans, this mixture was only marginally more efficient than when the components were used separately. ${ }^{(25)}$ In contrast to the previous study, Chu et al established that, for Streptococcus sanguis and Staphylococcus aureus, the mere addition of 25 percent by volume of Calyxl (a calcium hydroxide in saline paste; Otto and Co., Frankfurt, Germany) to Ledermix resulted in the conversion of a complete zone of inhibition, which was originally seen with Ledermix, to a zone of only partial inhibition. ${ }^{(26)}$ Taylor et al also concluded that there might not be any palpable synergistic or additive effects when combining two medicaments with strong antimicrobial activities. ${ }^{(25)}$

\section{Contraindications of corticosteroids}

Patients having systemic fungal infections or hypersensitivity to the drug must not be introduced to glucocorticoids. Special caution should be taken while using in patients with ulcerative co- 
litis, renal insufficiency, diabetes mellitus, diverticulitis, peptic ulcer, pyogenic infection, ocular herpes, osteoporosis, pregnancy, hypertension, acute psychosis, and a history of tuberculosis. ${ }^{(27)}$ Though there appear to be minimal drug interactions associated with the corticosteroids, the NSAIDs are not suggested to be used with them because of exaggerated gastrointestinal adverse effects.

Several medicaments have claimed, to date, to prevent and provide relief from painful exacerbations during root canal therapy. Among them, corticosteroids have shown successful results. Their anti-inflammatory activity is partly due to the inhibition of the fusion of lysozymes with their target membranes. ${ }^{(28)}$ With the aid of phospholipases, corticosteroids can also inhibit the release of free arachidonic acid from the phospholipids of cell membranes. Cortisone, owing to its effect on stabilizing the membranes, has a significant effect on pain. ${ }^{(28)}$ Though the exact mechanism is unknown, it is considered that this hormone might cause nerve hyperpolarization of the ones present in the area of inflammation or might augment the cyclic AMP production, which in turn, could trigger a protein kinase. This kinase might change the membrane permeability by causing phosphorylation of a protein constituent of the nerve cell membrane. ${ }^{(29)}$ This in return causes a hyperpolarization, discouraging the nerve impulse transmissions. Several eminent investigators have testified that corticosteroids placed into the root canal successfully control the pain. (16,30-32) however, some recent studies show contradictory results. ${ }^{(24)}$

\section{Disadvantages}

Though proven to be an excellent intracanal medicament, there are some drawbacks with corticosteroids in root canal therapy. While the concentration of inflammatory infiltrates in the periodontal space reduces by corticosteroids, they also interfere with protein synthesis. ${ }^{(33)}$ The outcome is that the infection becomes extensive, and the repair may be delayed or impaired.

Kim et al recorded another disadvantage when studying Ledermix paste. Regarding tooth discoloration, they reported that the possibility of discoloration was associated with the mode of application and the influence of sunlight on discoloration of mature teeth. ${ }^{(34)}$ The study outcomes demonstrated that the group treated with Ledermix produced dark grey-brown staining of teeth on exposure to sunlight after 12 weeks, but this was not seen on the specimens that were stored in the dark. Also, stains became worse when Ledermix pastes were used to fill the pulp chamber in comparison to teeth that were filled to the area below the cementoenamel junction (CEJ). ${ }^{(34)}$

\section{Conclusion}

Taking into consideration the innumerable studies and reviews done on steroids, we can safely conclude that systemic steroids are effective when used as an aide to, but not a replacement, for endodontic treatment in the reduction of post-treatment pain. In cases where patients present for treatment with moderate to severe pain, and in cases of necrotic pulp associated with periapical radiolucency, the systemic steroids have been proven successful. In addition, intraosseous and intraligamentary (PDL) injections significantly decrease postoperative endodontic pain. The pain-relieving efficacy of steroids and steroid-containing agents (such as Ledermix), when used as an intracanal medication, has been well documented.

\section{References}

1.Kumar A, Tamanna S, Iftekhar H. Intracanal medicaments - Their use in modern endodontics: A narrative review. J Oral Res Rev. 2019 Jul 1;11(2):94.

2.Paquette L, Legner M, Fillery ED, Friedman S. Antibacterial efficacy of chlorhexidine gluconate intracanal medication in vivo. J Endod. 2007 Jul 1;33(7):788-95.

3.Neelakantan P, Sanjeev K, Subbarao CV. Duration-dependent susceptibility of endodontic pathogens to calcium hydroxide and chlorhexidene gel used as intracanal medicament: an in vitro evaluation. Oral Surg Oral Med Oral Pathol Oral Radiol Endod. 2007 Oct;104(4):e138-41.

[4]

4.Cole TJ, Short KL, Hooper SB. The science of steroids. Semin Fetal Neonatal Med. 2019 Jun;24(3):170-175.

5.Ganong WF. Review of medical physiology. New York, McGraw-Hill, 2001:338-344.

6.Alexander RE, Throndson RR. A review of perioperative corticosteroid use in dentoalveolar surgery. Oral Surg Oral Med Oral Pathol Oral Radiol Endod. 2000 Oct 1;90(4):40615.

7.Haas DA. Emergency drugs. Dent Clin North Am. 2002 Oct 1;46(4):815-30.

8.Negm MM. Intracanal use of a corticosteroid-antibiotic 
compound for the management of posttreatment endodontic pain. Oral Surg Oral Med Oral Pathol Oral Radiol Endod. 2001 Oct 1;92(4):435-9.

9.Louwakul P, Lertchirakarn V. Incorporation of anti-inflammatory agent into calcium hydroxide pulp capping material: an in vitro study of physical and mechanical properties. Dent Mater J. 2012;31(1):32-9.

10.Kallali B, Singh K, Thaker V. Corticosteroids in dentistry. J Indian Acad Oral Med Radiol. 2011 Apr 1;23(2):128.

11.Ashwini P, Meena N, Santosh R, Kaiwan Anjali, Shetty Ashish, Naveen DN. Effect of long-term corticosteroids on dental pulp. World J Dent. 2010 Apr/Jun;1(1):17-20.

12.Hong D, Chen HX, Xue Y, Li DM, Wan XC, Ge R, et al. Osteoblastogenic effects of dexamethasone through upregulation of TAZ expression in rat mesenchymal stem cells. J Steroid Biochem Mol Biol. 2009 Aug;116(1-2):86-92.

13.Gotler M, Bar-Gil B, Ashkenazi M. Postoperative pain after root canal treatment: a prospective cohort study. Int J Dent. 2012;2012:310467.

14.Hargreaves KM, Goodis HE, Seltzer SS. Bender's dental pulp. Chicago, Quintessence Publishing, 2002:247.

15.Chong BS, Ford TP. The role of intracanal medication in root canal treatment. Int Endod J. 1992 Mar;25(2):97-106.

16.Ngeow WC, Lim D, Ahmad N. 66 Years of Corticosteroids in Dentistry: And We Are Still at a Cross Road? Corticosteroids. 2017 Dec 20. DOI: 10.5772/intechopen. 71540 17.Sharma N, Nikhil V, Gupta S. Effect of preoperative administration of steroid with different routes on post endodontic pain: a randomized placebo controlled clinical trial. Endodontology. 2015 Dec;27(2):107-2.

18.Athanassiadis M, Jacobsen N, Parashos P. The effect of calcium hydroxide on the steroid component of Ledermix ${ }^{\circledR}$ and Odontopaste ${ }^{\circledR}$. Int Endod J. 2011 Dec;44(12):1162-9.

19.Jindal V, Monga S, Jindal D. Comparative Evaluation of the Interappointment Pain by Using Different Intracanal Medicaments-An In Vivo Study. Dent J Adv Stud. 2019 Apr;7(01):019-22.

20.Balasubramaniam R, Jayakumar S. Antibiotics in endodontics-A concise review. IJADS. 2017;3(4):323-9.

21.Shamszadeh S, Shirvani A, Eghbal MJ, Asgary S. Efficacy of corticosteroids on postoperative endodontic pain: a systematic review and meta-analysis. J Endod. $2018 \mathrm{Jul}$ 1;44(7):1057-65.

22.Hargreaves K, Abbott PV. Drugs for pain management in dentistry. Aust Dent J. 2005 Dec;50:S14-22.

23.Dwi RS, Sundari AS, Liyana YH. Ledermix as Root Canal Medicament in Deciduous Teeth. J Appl Dent Med Sci. 2020;6:1.

24.Zanjir M, Sgro A, Lighvan NL, Yarascavitch C, Shah PS, da Costa BR, et al. Efficacy and Safety of Postoperative Medications in Reducing Pain after Nonsurgical Endodontic Treatment: A Systematic Review and Network Meta-analysis. J Endod. 2020 Oct;46(10):1387-1402.e4.

25.Taylor MA, Hume WR, Heithersay GS. Some effects of Ledermix ${ }^{\circledR}$ paste and Pulpdent ${ }^{\circledR}$ paste on mouse fibroblasts and on bacteria in vitro. Dent Traumatol. 1989 Dec;5(6):26673.

26.Chu FC, Leung WK, Tsang PC, Chow TW, Samaranayake LP. Identification of cultivable microorganisms from root canals with apical periodontitis following two-visit endodontic treatment with antibiotics/steroid or calcium hy- droxide dressings. J Endod. 2006 Jan 1;32(1):17-23.

27.Physicians' Desk Reference. 50th Edition Medical Consultant: Ronald Arky. Medical Economics Co., Montvale, NJ. 1996. J Med Chem. 1996, 39, 11, 2278.

28.Seltzer S, Naidorf IJ. Flare-ups in endodontics: II. Therapeutic measures. J Endod. 2004 Jul 1;30(7):482-8.

29.Kebabian JW, Greengard P. Dopamine-sensitive adenyl cyclase: possible role in synaptic transmission. Science. 1971 Dec 24;174(4016):1346-9.

30.Rosenteil-Heller P. Corticosteroids used in root canal fillings. Ten years of experience. Infor Dentaire. 1967;49:4949. 31.Langeland K, Langeland LK, Anderson DM. Corticosteroids in dentistry. Int Dent J. 1977 Sep 1;27(3):217-51.

32. Moskow A, Morse DR, Krasner P, Furst ML. Intracanal use of a corticosteroid solution as an endodontic anodyne. Oral Surg Oral Med Oral Pathol. 1984 Nov 1;58(5):600-4. 33.Perveen S, Yasmeen R, Yousaf A, Manzoor MA, Babar Z. Comparison of calcium hydroxide paste with kenacomb (corticosteroid-antibiotic paste) for postoperative endodontic pain in acute apical periodontitis: a randomized controlled trial. Pak Armed Forces Med J. 2014 Mar 31;64(1):39-45. 34.Kim ST, Abbott PV, McGinley P. The effects of Ledermix paste on discolouration of immature teeth. Int Endod J. 2000 May;33(3):233-7.

Cite this paper as:
Kar P, Varghese R, Agrawal N, Jhaveri H.
Steroid as an Intracanal Medicament: An Ad-
vanced Review. J Res Dent Maxillofac Sci. 2021;
6 (3) :47-51.

